\title{
Anthropometric and physical performance profiling does not predict professional contracts awarded in an elite Scottish soccer academy over a ten-year period.
}

\author{
CRAIG, T.P. and SWINTON, P.
}

This is an Accepted Manuscript version of the following article, accepted for publication in European Journal of Sport Science. CRAIG, T.P. and SWINTON, P. 2021. Anthropometric and physical performance profiling does not predict professional contracts awarded in an elite Scottish soccer academy over a ten-year period. European journal of sport science [online], 21(8), pages 1101-1110. Available from:

https://doi.org/10.1080/17461391.2020.1808079. It is deposited under the terms of the Creative Commons Attribution-NonCommercial License (http://creativecommons.org/licenses/by-nc/4.0/), which permits noncommercial re-use, distribution, and reproduction in any medium, provided the original work is properly cited. 
Anthropometric and physical performance profiling does not predict professional contracts awarded in an elite Scottish soccer academy over a ten-year period

THOMAS P. CRAIG ${ }^{1 *}$, PAUL SWINTON ${ }^{1 C}$

${ }^{1}$ School of Heath Sciences, Robert Gordon University, Aberdeen, AB10 7QG, UK

*Corresponding author: T.Craig3@RGU.ac.uk

Twitter:@ThoCra83

Linkedin: www.linkedin.com/in/thocra83 


\begin{abstract}
The purpose of this long-term retrospective analysis was to determine whether anthropometric and physical performance data could predict success in elite youth Scottish soccer players. Stature, body mass, sprint, jump and aerobic performance were collected from 512 players (U10 to U17) across a 10-year period. Players participated in an average of four profiling sessions (range: 1-14) and up to a maximum of three per year (August, December, and May) with standardisation applied to the surface, test order, time, and protocols. One hundred players were awarded professional contracts. Associations between variables were quantified with mixed-effects linear models. Prediction was assessed with least absolute shrinkage and selection operator (LASSO) regression developed on a training set ( $2 / 3$ data) and tested with proportion of error on a leave-out ( $1 / 3$ data) test set. Confidence intervals were obtained through bootstrap LASSO samples. A strong relative age bias was identified with $50 \%$ of successful players born in the first quarter of the year. Successful players were on average taller and performed better in sprint and jump tests $(p<0.05)$. However, effects were small and even when variables were combined, proportion of errors identified were similar to random guessing $(0.45[95 \% \mathrm{Cl}: 0.41-0.49])$. The results indicate that whilst successful players as youths demonstrate on average distinct anthropometric and physical profiles, these differences are unlikely to provide a reliable source to predict success within an already talented group. Practitioners should use data collected to guide exercise prescription but be aware of its substantive limitations in predicting success in isolation.
\end{abstract}

Key words: Talent identification; Relative age; Maturation; Talent development 


\section{Introduction}

Talent identification and development (TID) is a key driver within many soccer academy structures. Additionally, competition to identify and develop a country's most talented players becomes increasingly important under financial constraints. Talent identification in soccer frequently comprises anthropometric, psychological, physiological and soccer-specific skills profiling (Williams and Reilly, 2000). More holistic approaches may also consider sociological factors (Williams and Reilly, 2000) and the influence of maturation on trainability and development (Unnithan et al., 2012). However, despite the importance of TID there are limited data collected over the long-term to identify which factors can predict success in elite soccer academy graduates (Larkin and Reeves, 2018) and if individual factors such as physical performance are predictive (Bergkamp et al., 2019). The physical demands of soccer require in general a well-developed aerobic capacity, and anaerobic power to effectively engage in discrete game related tasks such as tackling, dribbling, sprinting, and accelerating (Deprez et al., 2015a). In addition, specific anthropometric profiles may provide advantages, particularly with regards to positional roles (Deprez et al., 2015a). Reflecting these large physical demands, it is well established that performance in physical tasks can differentiate between elite and non-elite youths in soccer (Slimani and Nikoladis, 2017). Additionally, study of anthropometric and physiological profiles of male soccer players have demonstrated that differences between elite and sub-elite cohorts and their playing positions can be observed as early as eight years old (Deprez et al., 2015a; Slimani and Nikoladis, 2017). However, it remains unclear whether data on individual players can be used to predict development and success during a soccer career.

A common method used to quantify success within a soccer academy is to identify player progression and ultimately the awarding of professional contracts. Lago-Penas et al., (2014) investigated the association of physical performance with continued academy progression across multiple age groups in elite Spanish academy players. A single physiological testing session conducted midway through the season identified that for certain positions successful players were significantly leaner and demonstrated greater jumping performance (Lago-Penas et al., 2014). Whilst the majority of measurements recorded identified no significant differences between successful and non-successful players, trends were identified for tests of muscular power with successful players tending to demonstrate higher values. The authors also identified positive associations between stature, mass and performance in muscular power tests (Lago-Penas et al., 2014). Therefore, it is possible that success may have been due to maturation status rather than physical performance per se.

A long-term prospective study investigating the associations between anthropometrics, physical performance and success was conducted by Höner and colleagues (2017) using nationwide data collected as part of a German TID program. The study involved 14,718 under 12 (U12) youth males representing the top $4 \%$ of soccer players. Anthropometrics and physical performance were assessed by measuring stature, body mass, $20 \mathrm{~m}$ sprint and agility times during a single baseline data collection session, with players followed up 8-10 years later. Data were also collected on skill performance quantified during dribbling, ball control and shooting drills. Eighty-nine of the players ultimately became professionals and 913 became semi-professionals. Univariate analyses of the U12 baseline data identified significant differences in mean values for height, weight and all physical and skill performance tests. Consistent rank order effects were identified for all variables with those obtaining professional contracts being taller, heavier and better performing in all tests at U12. However, the significant differences identified were influenced by the large sample size, with effects sizes calculated 
for all variables indicating negligible effects $\left(\eta^{2} \leq 0.016\right)$. To determine whether baseline assessment could predict success, complex logistic regression using performance tests to create latent variables was conducted. The model was able to explain only $25 \%$ of variation in the data, indicating that factors beyond those considered in the study were important in determining success. Importantly, this assessment of the predictive capability of the model is likely to be overestimated as the predictive model was built on the entire data set. For a realistic assessment of predictive capability crossvalidation with a "leave-out" set of data would be required to assess the degree of overfitting in the original assessment. An adjunct analysis was completed by Leyhr et al., (2018) with similar data collected from a single regional performance centre $(N=1,134)$ and included multiple assessments between $\mathrm{U} 12$ and $\mathrm{U} 15$ age groups. The results showed that performance in all tests improved nonlinearly across the four years and that successful players awarded professional or semi-professional contracts $(N=145)$ demonstrated significantly greater performances across all tests except $20 \mathrm{~m}$ sprint performance. Whilst regression models were able to explain substantial variance in performance tests, Leyhr et al., (2018) did not assess whether data collected across time points could be used to predict which players were awarded the contracts.

Le Gall et al., (2010) investigated anthropometric and physical differences of elite French youth soccer players (U14 - U16) over an 11-year period. Comparisons were made across the academy graduates who were subsequently grouped into those that played international soccer, those that were awarded professional contracts and those that achieved only amateur status (Le Gall et al., 2010). Out of a total of one hundred and fifty-three potential univariate statistical comparisons, only five identified significant differences between the groups. However, when the data were combined using multivariate statistics and spit by position, significant differences were identified for a larger range of variables. The authors concluded that whilst talent selection is likely influenced by a range of factors, their results demonstrated that fitness assessment data were important in determining whether already successful players would ultimately achieve the highest level of success (Le Gall et al., 2010). However, Le Gall et al., (2010) also concluded that further research was required to assess the extent to which anthropometric and physical profiling data could be used in selection. As with previous research, it is unclear the extent to which relatively small differences in a single aspect of distributions (e.g. means) with substantive standard deviations, can prospectively identity those that will become the most successful players.

A potential confounder when examining the relationship between physical performance and success within TID programs is the relative age effect (RAE). The phenomenon is common in youth sport where some players can be almost a year older than the youngest within a single year bracket, leading to intellectual, physiological and psychological differences (DeMeis and Stearns, 1992). Within the elite English system, the entry point to soccer academies demonstrate strong RAE bias and associated advantages with regards to size and physical performance criteria (Lovell et al., 2015; Cumming et al., 2018). The RAE bias has also been shown to continue throughout academy age ranges (Cumming et al., 2018). Whilst relative age was not shown to be influential in determining success in the large prospective study conducted by Honer et al., (2017), bias was already introduced into the sample with a skew towards players born in the first half of the year. Research conducted within Italian soccer has demonstrated that RAE is apparent at academy age and within non-elite adults but is less pronounced within the top division (Brustio et al., 2018). Given the inconsistency in findings regarding RAE and its relation to success in soccer, and its close connection with anthropometry and physical performance, its inclusion in further research investigating prediction of success is warranted. 
Given the financial restrictions that exist in many soccer academies there is often a focus on collecting inexpensive field-based physical tests to guide the TID process. Whilst the majority of previous research demonstrates that successful players will on average exhibit different anthropometric profiles and demonstrate greater physical performance, there is a paucity of information describing the ability to use the data and actually predict future success, rather than retrospectively explain variation in the data. Where such research has been conducted, there are often limitations including using all the data to build statistical models and not assessing overfitting through cross-validation (Höner et al., 2017). Therefore, the purpose of the present study was to determine using rigorous validation, whether retrospective data collected from an elite soccer academy over a longitudinal period could be used to predict success, and if so, which variables were most influential. It was hypothesised that whilst significant differences in anthropometry and physical performance would exist, these average differences would generate inaccurate predictions. 


\section{Methods}

A retrospect analysis of physical performance data comprising sprint, counter movement jump (CMJ) and aerobic endurance were assessed from 2006 to 2016 from an elite Scottish soccer academy whose players were involved in age matched competitive games across all playing positions. The data tracked 512 players, of which 100 were awarded professional status contacts, 362 were released and did not attain professional status at their parent or any other club, and 50 remained within the academy system at the final point of data collection. The data were collected as per the Club Academy Scotland criteria to "conduct research on players on a regular basis", with informed consent obtained by players and guardians through the contractual process. The retrospective analysis presented here received ethical approval by the institutional review board at the Robert Gordon University. Consistency was obtained across the entire data collection period by standardising playing surface (indoor sports hall), testing order, time of collection (seasonal observations on three evenings per year in August, December and May), consistent guidance regarding nutrition and hydration, and consistent involvement of a single practitioner overseeing the process and providing training to all support staff involved. Players that were injured did not participate and to increase validity, assessments conducted for the purpose of return to play, or for those on trial and not signed to the academy were excluded from the analysis. A total of 56 testing sessions were included over the ten years, comprising data from 512 players and a total of 2021 testing battery results. The average number of testing batteries completed by the players was 4 with a range of 1-14.

Given the consistency of the testing battery, the clear guidelines provided, and the regular inclusion of aspects of the tests in physical conditioning sessions, players were well familiarised with the testing process. The assessment battery comprised measurement of stature and body mass, followed by performance of CMJ with hands on hips, $20 \mathrm{~m}$ sprints (with intervals recorded at 5, 10 and $20 \mathrm{~m}$ ) and the YoYo Intermittent Recovery Test Level 1 (YYIR1). Stature was measured using a free-standing stadiometer (SECA Height Measure, Hamburg, Germany) and body mass measured using digital scales recording to $0.1 \mathrm{~kg}$ (SECA floor scale, Hamburg, Germany). A single measurement was recorded for stature then body mass due to time limitations. Participants then performed the same standardised warm-up comprising a progressive and intermittent dynamic structure (4x15 m jog, 12×15 m walking dynamic stretches incorporating all major muscle groups, 4x15 m leaps/bounds, 10x body mass squats, $4 \times 15 \mathrm{~m}$ progressive acceleration/decelerations). Physical testing began with three repetitions of the CMJ to a self-selected counter movement depth on an infra-red jump mat (MuscleLab IR Jump MAT, Ergotest, Langonsund, Norway). Repetitions that involved a pause during the counter movement, any flexion of the knees prior to landing, or landing outside the centre point of the jump mat were excluded, with additional trials allocated in order to obtain 3 successful jumps. The greatest jump height recorded was used for subsequent analyses. Immediately following the $\mathrm{CMJ}$, participants

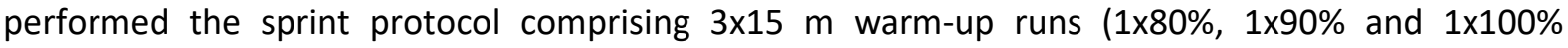
perceived maximum) followed by $3 \times 20 \mathrm{~m}$ maximum sprint efforts with timing gates recording split times to the nearest $0.01 \mathrm{~s}$ (Speed Trap Timing, Brower Timing Systems, Utah, USA). Each sprint was separated with a recovery period of at least 1:10, with the start line located $1 \mathrm{~m}$ behind the initial timing gate. Participants initiated sprints with one foot self-selected in front of the other. The sprint that recorded the lowest time to complete the $20 \mathrm{~m}$ was selected for further analysis along with the associated $5 \mathrm{~m}$ and $10 \mathrm{~m}$ split times. A ten-minute recovery period was provided between sprints and the YYIR1 assessment using recommended criteria for test termination. Appropriate test validity and reliability for all equipment and protocols adopted in the testing battery have been established 
previously with similar age groups within soccer (Bosquet et al., 2009; Paul and Nassis, 2015, Dugdale et al., 2019; Deprez et al., 2015b).

\section{Statistical Analysis}

Existence of a relative age bias was assessed by comparing the number of births across the four yearly quarters using a $\chi^{2}$ test. Mixed-effect linear regression models were performed for each of the physical tests. Chronological age was entered as a covariate and standardised by subtracting the minimum value of 10 to assist interpretation. Relative age was included as a four-level factor variable according to the quarter of birth, and player success was entered as a binary variable according to whether or not a player received a professional contract. A random intercept and slope for age was included for each player. The association of covariates with player success were then modelled using least absolute shrinkage and selection operator (LASSO) logistic regression with multiple imputation (Zhang, 2016) and bootstrapping $(n=10,000)$. Relative age and all anthropometric and physical performance variables collected across the testing battery were included in the models. Optimal LASSO penalty was obtained via tenfold cross-validation. Continuous regression inputs were centered and scaled by dividing values by two standard deviations to facilitate comparisons with binary predictors and to assist with model interpretation (Gelman, 2008). Finally, the ability to predict player success was assessed through bootstrap LASSO models and pairing individual testing profiles of successful players with an equal number of testing profiles from non-successful players. Each LASSO bootstrap model was trained on two thirds of the data and then tested on a leave-out one third test set. Predictive capability was assessed by quantifying the proportion of error and $95 \%$ confidence interval (CI). All analyses were performed using the R programming language (version 3.5.3). 


\section{Results}

The breakdown of testing batteries performed across the different age groups are presented in table 2 , with the results showing a relatively even split across the under 11 to under 17 age groups. Of the 100 players awarded a professional contract, the age group at which these players entered the Academy was also relatively evenly split across under 11's to under 17's (Table 2).

A relative age bias was identified across the entire group of 512 players, with the proportion of births across the four yearly quarters equal to: $\mathrm{Q} 1=0.37 ; \mathrm{Q} 2=0.28 ; \mathrm{Q} 3=0.22$; and $\mathrm{Q}=0.13\left(\chi_{3}^{2}=64\right.$, $\mathrm{p}<0.001)$. A stronger relative age bias was identified across the 100 players awarded a professional contract with the proportion of births across the four yearly quarters equal to: $\mathrm{Q} 1=0.50 ; \mathrm{Q} 2=0.26$; $\mathrm{Q} 3=0.20$; and $\mathrm{Q}=0.05\left(\chi_{3}^{2}=40.9, \mathrm{p}<0.001\right)$.

The effects of chronological age, player success and relative age on average performance across the physical measures were assessed via mixed-effects linear regression models (Table 3). Linear effects of chronological age indicated that for the majority of variables an average improvement of between 2 to $10 \%$ was achieved each year. Successful players were on average taller $(p<0.05)$, were faster over $20 \mathrm{~m}(p<0.01)$ and demonstrated greater vertical jump performance $(p<0.01)$. However, despite significant differences in average values, there was considerable variation and overlap between successful and non-successful players (Figure 1). Regression coefficients for relative age indicated a trend towards players born in the first quarter of the year being taller, heavier and better performing in physical tests. However, the majority of coefficients were non-significant due to large standard errors.

Associations between predictor variables and success were quantified on imputed data sets comprising a total of 1850 testing battery. Mixed-effects binomial regression models controlling for age were completed for each predictor (Table 4), with significant associations identified for relative age (Q3 vs. Q1: $p<0.05$ and $Q 4$ vs. Q1: $p<0.001)$, height $(p<0.05), 20 \mathrm{~m}$ sprint time $(p<0.05)$, CMJ height $(p<0.001)$, YYIR1 level $(p<0.05)$ and YYIR1 distance $(p<0.05)$. All predictors were then simultaneously included in LASSO regression with bootstrap samples. The most frequently included predictors comprised age, relative age (Q4 vs. Q1), stature and CMJ performance. However, associations between predictors and contract success were low, with the greatest reduced odds ratio equal to 0.73 when comparing relative age (Q4 vs. Q1), and the greatest increased odds ratio equal to 1.2 for CMJ. The ability to predict whether a given physical profile would lead to success was assessed by calculating the proportion of error on the LASSO training set ( $2 / 3$ of data) and testing set ( $1 / 3$ of data), with bootstrap samples to obtain Cls. With error of 0.5 obtained with random guessing, results demonstrated effectively no ability to predict success with $0.43(0.39-0.47)$ error proportion obtained with the training set and $0.45(0.41-0.49)$ error proportion obtained with the test set. 


\section{Discussion}

The present study conducted a retrospective analysis of anthropometric and physical performance data collected across a ten-year period with male youths from an elite Scottish soccer academy. The analysis featured the modelling of routinely collected data to determine whether successful players awarded a professional contract were different on average from non-successful players, and given the paucity of previous information, to assess whether any differences found had the potential to reliably predict success. The analyses demonstrated strong relative age bias and consistent differences in average values of anthropometric and physical performance between successful players awarded a professional contract and non-successful players. However, predictive modelling conducted under a rigorous cross-validation process was unable to distinguish between successful and non-successful players and prediction was shown to perform similarly to random guessing. The results demonstrate that whilst small average differences exist and indicate an advantage in anthropometric and physical performance variables for successful versus non-successful players, the substantial variation and overlap is such that prediction with the data on a case by case basis is ineffective.

The results presented here describing significant differences and trends of greater physical performance of successful versus non-successful youth soccer players are consistent with previous research defining success based on either progression through an academy system (Lago-Penas et al., 2014; Hirose and Seki, 2015; Deprez et al., 2015b; Leyhr et al., 2018) or being awarded professional contracts (Deprez et al., 2015b; Martinez-Santos et al., 2016 ; Honer et al., 2017; Los Arcos and Martins, 2018). However, whilst the research base has clearly established that average differences are likely to exist, multiple factors limit the predictive capability and associated errors when using anthropometric or physical performance data. In the largest cohort study conducted by Höner et al., (2017) with 14,718 youth players, it was identified that successful players performed better on average in all physical and skill performance tests. However, effect sizes associated with each test in isolation were small $(\leq 0.016)$ and would therefore be unable to effectively predict. When data across multiple tests were combined, Höner et al., (2017) demonstrated that more effective models could be developed. However, percentage of explained variance was only $25 \%$ with multivariate models and therefore predictive capability would also be expected to be ineffective. Additionally, models presented by Höner et al., (2017) were developed using the entire data set and therefore likely to overfit the data. In the present study LASSO regression models that seek to shrink coefficients to zero and develop parsimonious solutions were used. In addition, models were developed on a training set and then tested on a leave-out set to assess potential issues with overfitting. Collectively, the results from the present study highlight the limited ability of factors that demonstrate small average differences to predict successful players, either individually or in combination through multivariate models.

Whilst the results presented here demonstrate the inability for anthropometric and physical performance data to predict success within an already talented group of individuals, physical development remains important in the TID pathway (Williams and Reilly, 2000). Research has established clear differences between elite and sub-elite cohorts (Deprez et al., 2015a; Slimani and Nikoladis, 2017) and it is argued that players that do not exhibit sufficient physical capability are unlikely to progress through an academy system (Deprez et al., 2015b). In the present study, the single most influential factor predicting success was relative age, with the largest difference identified between those born in the first quarter and last quarter of the year. The finding of a strong RAE is 
consistent with previous research (Jimenez and Pain, 2008; Lovell et al., 2015; Adronikos et al., 2016; Mann and van Ginnekan, 2017; Cumming et al., 2018) and of significant concern when attempting to identify and develop a countries most talented players (Doyle and Bottomley, 2019). Underrepresentation of players born in the later months of the year represents a loss and inefficiency in TID (Jimenez and Pain, 2008), even if the RAE becomes less apparent at the level of senior squads (Brustio et al., 2018; Ford and Williams, 2011). A range of approaches have been implemented to reduce the RAE, with "bio-banding" activities been shown to have a positive influence within youth soccer (Cumming et al., 2017; Abbott et al., 2019). Bio-banding involves grouping players according to maturation status or biological age to support late maturing players in terms of physical challenge and early maturating players in terms of technical and psychological competencies (Cumming et al., 2017). Empirical data from technical variables within bio-banding has revealed shorter passing sequences and more dribbles for late developers whilst providing early developers increased tackles and an avoidance of long passes (Abbott et al., 2019). However, whilst the manipulation of age categories in groups of 3 or 6 months may have the potential to reduce the REA (Sierra-Diaz et al., 2017), such a system may create financial burdens that would be challenging for many TID programs. In contrast, educating scouts not to focus on competitive success, but instead, to focus on technical capabilities, skills and physical capabilities with greater context of relative age may reduce selection bias (Adronikos et al., 2016; Mann and van Ginnekan, 2017). Practitioners and TID managers should consider the implementation of competitive bio banding opportunities for their clubs as part of their long term TID process. Competition with external organisations can ensure developmental opportunities as observed by Abbott et al., (2019) whilst the involvement of scouts and decision makers in these bio-banded environments could potentially enhance coach education (Adronikos et al., 2016), but certainly allow for players to be assessed in the absence of a negative REA context.

In a recent review of methodological issues related to TID research in soccer, Bergkamp et al., (2019) identified a number of challenges that are relevant to the present study. Bergkamp et al., (2019) argued that whilst research such as that presented here can increase understanding of important TID factors, determining success in terms of progression or attainment of professional contracts is limited and research should focus on the extent to which explanatory factors can predict actual soccer performance. However, at present no single objective measure exists and the development of a valid and reliable measure of individual soccer performance is likely to be a complex process to account for multiple non-linearly interacting components and context-specific elements in relation to opposition, country of investigation, different positions and tactics employed (Baker et al., 2015; Davids et al., 2013; Franks et al, 1999). Bergkamp et al., (2019) also identified the limitation of building predictive models focusing solely on anthropometry and physical performance, and the need to include more soccer specific explanatory measures. Whilst the development of such measures is unlikely to be as challenging as that to develop a criterion measure of soccer performance, additional research is required to determine valid and reliable tests and processes such as whether these measures should be collected longitudinally and then suitably transformed for analyses. An additional limitation of the present research inherent in other TID studies include the restriction of the analysis to a relatively homogenous population. Analysis of the relative age bias in the present study noted substantial departures from that seen in the wider population indicating a strong initial selection bias and as a result a restricted range for predictors to identify successful and non-successful players (Bergkamp et al., 2019). This limitation in future can be avoided by including data from a wider population, however, this would also require an effective criterion measure of soccer performance to incorporate the additional data. 


\section{Practical implications}

The present study employed a low-cost method that could feasibly be used by a soccer academy to use data routinely collected to inform their TID programme. By building up a bank of information and categorising players based on their success, there is the potential to build models that could be used to make predictions of future prospects whilst continually updating models with the subsequent generation of new data. Previous research has demonstrated that successful players from soccer academies tend to exhibit on average different anthropometric and physical performance profiles, suggesting that this information which can be collected at low cost could be used as a source for predictions. However, the current study which assessed this hypothesis in a rigorous statistical manner has demonstrated that this is unlikely to lead to effective results and was very similar to random guessing. Using similar robust statistical procedures, researchers and practitioners within soccer academies that are continually collecting data should assess whether accurate predictions can be made combining data across a holistic range of dimensions including physiological, technical, psychological, tactical skills and expertise from technical coaches.

The finding of a very strong RAE is an important one, particularly for soccer academies that operate under financial restraints. Academies should consider processes such as coach and scout education programmes to reduce the negative impacts of controllable factors such as the RAE. Economy of TID programmes is an important consideration and enhanced coach education together with enhanced player development opportunities such as bio-banding could reduce unnecessary release of players that are at the point, underdeveloped as opposed to untalented.

Whilst the present study demonstrated the limitations of using anthropometric and physical performance profiling data to predict who will become a successful player, the information is still likely to be an important part of the talent development process with data being used to assist the creation and individual tailoring of physical training. Additionally, the creation of normative data can be used by soccer academies to monitor for continual progression of physical capabilities as advances in exercise science and increased availability of technology occurs.

\section{Declaration of Interests}

Funding: No external funding was used for this work. 


\section{References}

Abbott, W., Williams, S., Brickley, G. and Smeeton, N.J., 2019. Effects of Bio-Banding upon Physical and Technical Performance during Soccer Competition: A Preliminary Analysis. Sports, 7(8), p.193.

Andronikos, G., Elumaro, A.I., Westbury, T. and Martindale, R.J., 2016. Relative age effect: implications for effective practice. Journal of sports sciences, 34(12), pp.1124-1131.

Baker J, Wattie N, Schorer J. Defning expetise: a taxonomy for researchers in skill acquisition and expertise. In: Baker J, Farrow D, editors. Routlegde handbook of sport expertise. London: Routledge; 2015. p. 183-95.

Bergkamp, T.L., Niessen, A.S.M., den Hartigh, R.J., Frencken, W.G. and Meijer, R.R., 2019. Methodological issues in soccer talent identification research. Sports Medicine, 49(9), pp.1317-1335.

Bosquet, L., Berryman, N. and Dupuy, O., 2009. A comparison of 2 optical timing systems designed to measure flight time and contact time during jumping and hopping. The Journal of Strength \& Conditioning Research, 23(9), pp.2660-2665.

Brustio, P.R., Lupo, C., Ungureanu, A.N., Frati, R., Rainoldi, A. and Boccia, G., 2018. The relative age effect is larger in Italian soccer top-level youth categories and smaller in Serie A. PloS one, 13(4).

Cumming, S.P., Lloyd, R.S., Oliver, J.L., Eisenmann, J.C. and Malina, R.M., 2017. Bio-banding in sport: applications to competition, talent identification, and strength and conditioning of youth athletes. Strength \& Conditioning Journal, 39(2), pp.34-47.

Cumming, S.P., Searle, C., Hemsley, J.K., Haswell, F., Edwards, H., Scott, S., Gross, A., Ryan, D., Lewis, J., White, P. and Cain, A., 2018. Biological maturation, relative age and self-regulation in male professional academy soccer players: A test of the underdog hypothesis. Psychology of Sport and Exercise, 39, pp.147-153.

Davids, K., Araújo, D., Vilar, L., Renshaw, I. and Pinder, R., 2013. An ecological dynamics approach to skill acquisition: implications for development of talent in sport. Talent Development and Excellence, 5(1), pp.21-34.

DeMeis, J.L. and Stearns, E.S., 1992. Relationship of school entrance age to academic and social performance. The Journal of Educational Research, 86(1), pp.20-27. 
Deprez, D., Fransen, J., Boone, J., Lenoir, M., Philippaerts, R. and Vaeyens, R., 2015a. Characteristics of high-level youth soccer players: variation by playing position. Journal of Sports Sciences, 33(3), pp.243-254.

Deprez, D.N., Fransen, J., Lenoir, M., Philippaerts, R.M. and Vaeyens, R., 2015b. A retrospective study on anthropometrical, physical fitness, and motor coordination characteristics that influence dropout, contract status, and first-team playing time in high-level soccer players aged eight to eighteen years. The Journal of Strength \& Conditioning Research, 29(6), pp.1692-1704.

Doyle, J.R. and Bottomley, P.A., 2019. The relative age effect in European elite soccer: A practical guide to Poisson regression modelling. PloS one, 14(4).

Dugdale, J.H., Arthur, C.A., Sanders, D. and Hunter, A.M., 2019. Reliability and validity of field-based fitness tests in youth soccer players. European journal of sport science, 19(6), pp.745-756.

Franks, A., Williams, A.M., Reilly, T. and Nevill, A., 1999. Talent identification in elite youth soccer players: Physical and physiological characteristics. Journal of Sports Sciences, 17(10), p.812.

Ford, P.R. and Williams, M.A., 2011. No relative age effect in the birth dates of award-winning athletes in male professional team sports. Research quarterly for exercise and sport, 82(3), pp.570573.

Gelman, A., 2008. Scaling regression inputs by dividing by two standard deviations. Statistics in medicine, 27(15), pp.2865-2873.

Hirose, N. and Seki, T., 2016. Two-year changes in anthropometric and motor ability values as talent identification indexes in youth soccer players. Journal of science and medicine in sport, 19(2), pp.158-162.

Höner, O., Leyhr, D. and Kelava, A., 2017. The influence of speed abilities and technical skills in early adolescence on adult success in soccer: A long-term prospective analysis using ANOVA and SEM approaches. PloS one, 12(8).

Lago-Peñas, C., Rey, E., Casáis, L. and Gómez-López, M., 2014. Relationship between performance characteristics and the selection process in youth soccer players. Journal of human kinetics, $40(1)$, pp.189-199. 
Larkin, P. and Reeves, M.J., 2018. Junior-elite football: time to re-position talent identification? Soccer \& Society, 19(8), pp.1183-1192.

Le Gall, F., Carling, C., Williams, M. and Reilly, T., 2010. Anthropometric and fitness characteristics of international, professional and amateur male graduate soccer players from an elite youth academy. Journal of science and medicine in sport, 13(1), pp.90-95.

Leyhr, D., Kelava, A., Raabe, J. and Höner, O., 2018. Longitudinal motor performance development in early adolescence and its relationship to adult success: An 8-year prospective study of highly talented soccer players. PloS one, 13(5).

Los Arcos, A. and Martins, J., 2018. Physical Fitness Performance of Young Professional Soccer Players Does Not Change During Several Training Seasons in a Spanish Elite Reserve Team: Club Study, 1996-2013. The Journal of Strength \& Conditioning Research, 32(9), pp.2577-2583.

Lovell, R., Towlson, C., Parkin, G., Portas, M., Vaeyens, R. and Cobley, S., 2015. Soccer player characteristics in English lower-league development programmes: the relationships between relative age, maturation, anthropometry and physical fitness. PloS one, 10(9).

Jiménez, I.P. and Pain, M.T., 2008. Relative age effect in Spanish association football: Its extent and implications for wasted potential. Journal of sports sciences, 26(10), pp.995-1003.

Mann, D.L. and van Ginneken, P.J., 2017. Age-ordered shirt numbering reduces the selection bias associated with the relative age effect. Journal of sports sciences, 35(8), pp.784-790.

Martinez-Santos, R., Castillo, D. and Los Arcos, A., 2016. Sprint and jump performances do not determine the promotion to professional elite soccer in Spain, 1994-2012. Journal of sports sciences, 34(24), pp.2279-2285.

Paul, D.J. and Nassis, G.P., 2015. Physical fitness testing in youth soccer: Issues and considerations regarding reliability, validity, and Sensitivity. Pediatric exercise science, 27(3), pp.301-313.

Sierra-Díaz, M.J., González-Víllora, S., Pastor-Vicedo, J.C. and Serra-Olivares, J., 2017. Soccer and relative age effect: A walk among elite players and young players. Sports, 5(1), p.5. 
Slimani, M. and Nikolaidis, P.T., 2017. Anthropometric and physiological characteristics of male Soccer players according to their competitive level, playing position and age group: a systematic review. J Sports Med Phys Fitness, pp.07950-6.

Unnithan, V., White, J., Georgiou, A., Iga, J., \& Drust, B. (2012). Talent identification in youth soccer. Journal of sports sciences, 30(15), 1719-1726.

Williams, A.M. and Reilly, T., 2000. Talent identification and development in soccer. Journal of sports sciences, 18(9), pp.657-667.

Zhang, Z., 2016. Multiple imputation with multivariate imputation by chained equation (MICE) package. Annals of translational medicine, 4(2). 
Table 1: Testing battery data across age groups (mean \pm standard deviation)

\begin{tabular}{lccccccc}
\hline Test & U10s & U11s & U12s & U13s & U14s & U15s & U17s \\
\hline Stature $(\mathrm{cm})$ & $136.1 \pm 4.7$ & $143.2 \pm 6.0$ & $147.9 \pm 6.6$ & $155.4 \pm 7.6$ & $162.8 \pm 7.9$ & $171.0 \pm 7.1$ & $175.2 \pm 6.3$ \\
Mass $(\mathrm{kg})$ & $31.5 \pm 3.7$ & $35.5 \pm 4.9$ & $38.5 \pm 5.5$ & $43.8 \pm 7.0$ & $51.3 \pm 7.5$ & $59.0 \pm 8.1$ & $65.7 \pm 7.6$ \\
BMI (kgm $\left.{ }^{-2}\right)$ & $17.0 \pm 1.3$ & $17.2 \pm 1.5$ & $17.5 \pm 1.5$ & $18.2 \pm 1.6$ & $19.2 \pm 1.6$ & $20.1 \pm 2.0$ & $21.3 \pm 1.9$ \\
$5 \mathrm{~m}$ Time $(\mathrm{s})$ & $1.14 \pm .07$ & $1.13 \pm .07$ & $1.11 \pm .07$ & $1.08 \pm .07$ & $1.06 \pm .07$ & $1.04 \pm .07$ & $1.03 \pm .07$ \\
$10 \mathrm{~m}$ Time $(\mathrm{s})$ & $2.02 \pm .11$ & $2.00 \pm .10$ & $1.96 \pm .07$ & $1.91 \pm .09$ & $1.85 \pm .09$ & $1.81 \pm .11$ & $1.78 \pm .11$ \\
$20 \mathrm{~m}$ Time $(\mathrm{s})$ & $3.63 \pm .20$ & $3.57 \pm .16$ & $3.50 \pm .16$ & $3.39 \pm .15$ & $3.26 \pm .16$ & $3.14 \pm .14$ & $3.07 \pm .14$ \\
CMJ (cm) & $20.0 \pm 3.6$ & $23.4 \pm 4.3$ & $25.5 \pm 4.6$ & $27.1 \pm 4.4$ & $30.8 \pm 5.3$ & $33.8 \pm 4.9$ & $36.3 \pm 5.0$ \\
YYIR1 Level & $15.1 \pm .96$ & $15.7 \pm 1.3$ & $16.5 \pm 1.4$ & $17.3 \pm 1.5$ & $17.9 \pm 1.5$ & $18.9 \pm 1.6$ & $19.1 \pm 1.8$ \\
YYIR1 Distance $(\mathrm{m})$ & $822 \pm .279$ & $1037 \pm 377$ & $1273 \pm 425$ & $1524 \pm 449$ & $1728 \pm 471$ & $2041 \pm 512$ & $2114 \pm 573$ \\
\hline
\end{tabular}

Table 2: Summary of testing observations and number of players ultimately awarded a professional contract across age groups

\begin{tabular}{lccccccc}
\hline Age group & U10s & U11s & U12s & U13s & U14s & U15s & U17s \\
\hline Number of testing & 68 & 378 & 312 & 305 & 339 & 287 & 332 \\
observations (\%) & $(3.4 \%)$ & $(18.7 \%)$ & $(15.4 \%)$ & $(15.1 \%)$ & $(16.8 \%)$ & $(14.2 \%)$ & $(16.4 \%)$ \\
$\begin{array}{l}\text { Number of players from cohort } \\
\text { awarded professional contract }\end{array}$ & 3 & 19 & 11 & 18 & 14 & 13 & 22 \\
\hline
\end{tabular}

Table 3: Mixed-effects linear regression models (regression coefficients [standard errors]) quantifying effects of chronological age, relative age and player success on physical factors assessed.

\begin{tabular}{lllllll}
\hline $\begin{array}{l}\text { Independent } \\
\text { Variable }\end{array}$ & Intercept & Age & Successful & $\begin{array}{l}\text { Relative Age } \\
\text { Q2 }\end{array}$ & $\begin{array}{l}\text { Relative Age } \\
\text { Q3 }\end{array}$ & $\begin{array}{l}\text { Relative Age } \\
\text { Q4 }\end{array}$ \\
\hline Stature $(\mathrm{cm})$ & $140^{* * *}$ & $5.2^{* * *}$ & $1.8^{*}$ & -.97 & $-1.1^{*}$ & -2.2 \\
& {$[0.71]$} & {$[.11]$} & {$[.82]$} & {$[.71]$} & {$[.78]$} & {$[.95]$} \\
Mass $(\mathrm{kg})$ & $31.1^{* * *}$ & $4.9^{* * *}$ & .68 & -.14 & -1.3 & -.76 \\
& {$[.54]$} & {$[.09]$} & {$[.77]$} & {$[.65]$} & {$[.73]$} & {$[.88]$} \\
BMI $\left(\mathrm{kgm}^{-2}\right)$ & $16.6^{* * *}$ & $.65^{* * *}$ & -.01 & .09 & -.22 & .12 \\
& {$[.14]$} & {$[.02]$} & {$[.19]$} & {$[.17]$} & {$[.18]$} & {$[.22]$} \\
5 m Time (s) & $1.2^{* * *}$ & $-0.02^{* * *}$ & -.007 & .000 & .008 & .008 \\
& {$[.005]$} & {$[.001]$} & {$[.005]$} & {$[.006]$} & {$[.006]$} & {$[.008]$} \\
$10 \mathrm{~m}$ Time (s) & $2.0^{* * *}$ & $-.04^{* * *}$ & -.01 & -.01 & .01 & .007 \\
& {$[.0083]$} & {$[.001]$} & {$[.01]$} & {$[.009]$} & {$[.009]$} & {$[.01]$} \\
$20 \mathrm{~m}$ Time (s) & $3.7^{* * *}$ & $-.09^{* * *}$ & $-.04^{* *}$ & -.009 & .005 & .01 \\
& {$[.01]$} & {$[.002]$} & {$[.01]$} & {$[.02]$} & {$[.02]$} & {$[.02]$} \\
CMJ (cm) & $21.3^{* * *}$ & $2.2^{* * *}$ & $1.2^{* *}$ & -.08 & -.76 & -1.1 \\
& {$[.38]$} & {$[.07]$} & {$[0.50]$} & {$[.43]$} & {$[.47]$} & {$[.60]$} \\
YYIR1 Level & $14.8^{* * *}$ & $.74^{* * *}$ & .12 & -.28 & -.24 & -.38 \\
& {$[.11]$} & {$[.02]$} & {$[.15]$} & {$[.14]$} & {$[.15]$} & {$[.19]$} \\
YYIR1 Distance $(\mathrm{m})$ & $737^{* * *}$ & $233^{* * *}$ & 37.4 & $-85.6 *$ & -75.7 & -108 \\
& {$[34.2]$} & {$[6.4]$} & {$[45.2]$} & {$[42.9]$} & {$[47.1]$} & {$[57.8]$} \\
\hline
\end{tabular}

Chronological age was centred by subtracting 10 from each age year. Therefore, intercept quantifies the average value for U10 players that did not obtain a professional contract born in the first quarter of the year. ${ }^{*} p<0.05,{ }^{* *} p<0.01,{ }^{* * *} p<0.001$. 
Table 4: Mixed-effects univariate logistic and multivariate LASSO logistic regression models.

\begin{tabular}{|c|c|c|c|c|c|c|c|}
\hline \multirow[t]{2}{*}{ Predictor } & \multirow{2}{*}{$\begin{array}{c}\text { Logistic } \\
\text { Regression } \\
\text { Coefficient } \\
{[\mathrm{SE}]} \\
\end{array}$} & \multicolumn{2}{|c|}{$\begin{array}{l}\text { LASSO Regression } \\
\text { [Full data] }\end{array}$} & \multicolumn{2}{|c|}{$\begin{array}{l}\text { LASSO Regression } \\
\text { [U10-U13] }\end{array}$} & \multicolumn{2}{|c|}{$\begin{array}{l}\text { LASSO Regression } \\
\text { [U14 - U17] }\end{array}$} \\
\hline & & $\begin{array}{c}\text { Coefficient } \\
{[95 \% \mathrm{Cl}]}\end{array}$ & $\%$ & $\begin{array}{l}\text { Coefficient } \\
{[95 \% \mathrm{Cl}]}\end{array}$ & $\%$ & $\begin{array}{c}\text { Coefficient } \\
{[95 \% \mathrm{Cl}]}\end{array}$ & $\%$ \\
\hline Intercept & ---- & $-.72+[-.82--.60]$ & 100 & $-.88+[-1.3--0.48]$ & 100 & $-.70+[-.94--.45]$ & 100 \\
\hline Age & ---- & $.12+[.01-.23]$ & 99 & $.05[0-.26]$ & 74 & $.10[0-.26]$ & 90 \\
\hline $\begin{array}{l}\text { Relative } \\
\text { Age Q2 }\end{array}$ & $\begin{array}{l}-.13 \\
{[.13]}\end{array}$ & $0[-.03-0]$ & 4 & $0[-.44-0]$ & 5 & $0[-.03-0]$ & 6 \\
\hline $\begin{array}{l}\text { Relative } \\
\text { Age Q3 }\end{array}$ & $\begin{array}{l}-.33^{*} \\
{[.14]}\end{array}$ & $0[-.22-0]$ & 28 & $0[0-0]$ & 4 & $-.04[-.39-0]$ & 61 \\
\hline $\begin{array}{l}\text { Relative } \\
\text { Age Q4 }\end{array}$ & $\begin{array}{c}-1.2 * * * \\
{[.24]}\end{array}$ & $-.31[-.86-0]$ & 94 & $-.14[.91-0]$ & 72 & $-.28[-.83-0]$ & 85 \\
\hline Height & $\begin{array}{l}.40^{*} \\
{[.21]}\end{array}$ & $.13[0-.54]$ & 70 & $.04[0-.88]$ & 56 & $0[0-.37]$ & 30 \\
\hline Weight & $\begin{array}{l}.30 \\
{[.19]}\end{array}$ & $0[0-.36]$ & 41 & $.26[0-1.2]$ & 75 & $0[0-0]$ & 2 \\
\hline BMI & $\begin{array}{l}.12 \\
{[.13]}\end{array}$ & $0[0-.15]$ & 10 & $0[0-.15]$ & 6 & $0[0-0]$ & 7 \\
\hline $5 \mathrm{~m}$ Time & $\begin{array}{l}-.16 \\
{[.13]}\end{array}$ & $0[-.04-0]$ & 5 & $0[0-.20]$ & 13 & $0[-.27-0]$ & 21 \\
\hline 10m Time & $\begin{array}{l}-.11 \\
{[.14]}\end{array}$ & $0[0-0]$ & 1 & $0[0-.20]$ & 10 & $0[-.02-0]$ & 4 \\
\hline 20m Time & $\begin{array}{l}-.36^{*} \\
{[.18]}\end{array}$ & $0[-.34-0]$ & 43 & $0[0-0]$ & 1 & $-.60+[-1.1--.08]$ & 99 \\
\hline $\mathrm{CMJ}$ & $\begin{array}{l}.44 * * * \\
{[.15]}\end{array}$ & $.19[0-0.47]$ & 89 & $0[0-.50]$ & 41 & $.01[0-.40]$ & 54 \\
\hline $\begin{array}{l}\text { YoYo } \\
\text { Level }\end{array}$ & $\begin{array}{l}.31^{*} \\
{[.15]}\end{array}$ & $0[0-0.36]$ & 38 & $0[0-.04]$ & 4 & $.01[0-.44]$ & 52 \\
\hline $\begin{array}{l}\text { YoYo } \\
\text { Distance }\end{array}$ & $\begin{array}{l}.33^{*} \\
{[.15]}\end{array}$ & $.02[0-0.39]$ & 55 & $0[0-.19]$ & 9 & $0[0-.35]$ & 33 \\
\hline
\end{tabular}

$\%$ represents the percentage of bootstrap samples for which the predictor was included in the model [non-zero regression coefficient]. ${ }^{*} p<0.05,{ }^{* * *} p<0.001$. +LASSO models not including 0 in either 0.025 or 0.975 quantile. 
Figure 1: Distribution of countermovement jump (CMJ) performances across age groups between successful and non-successful players.

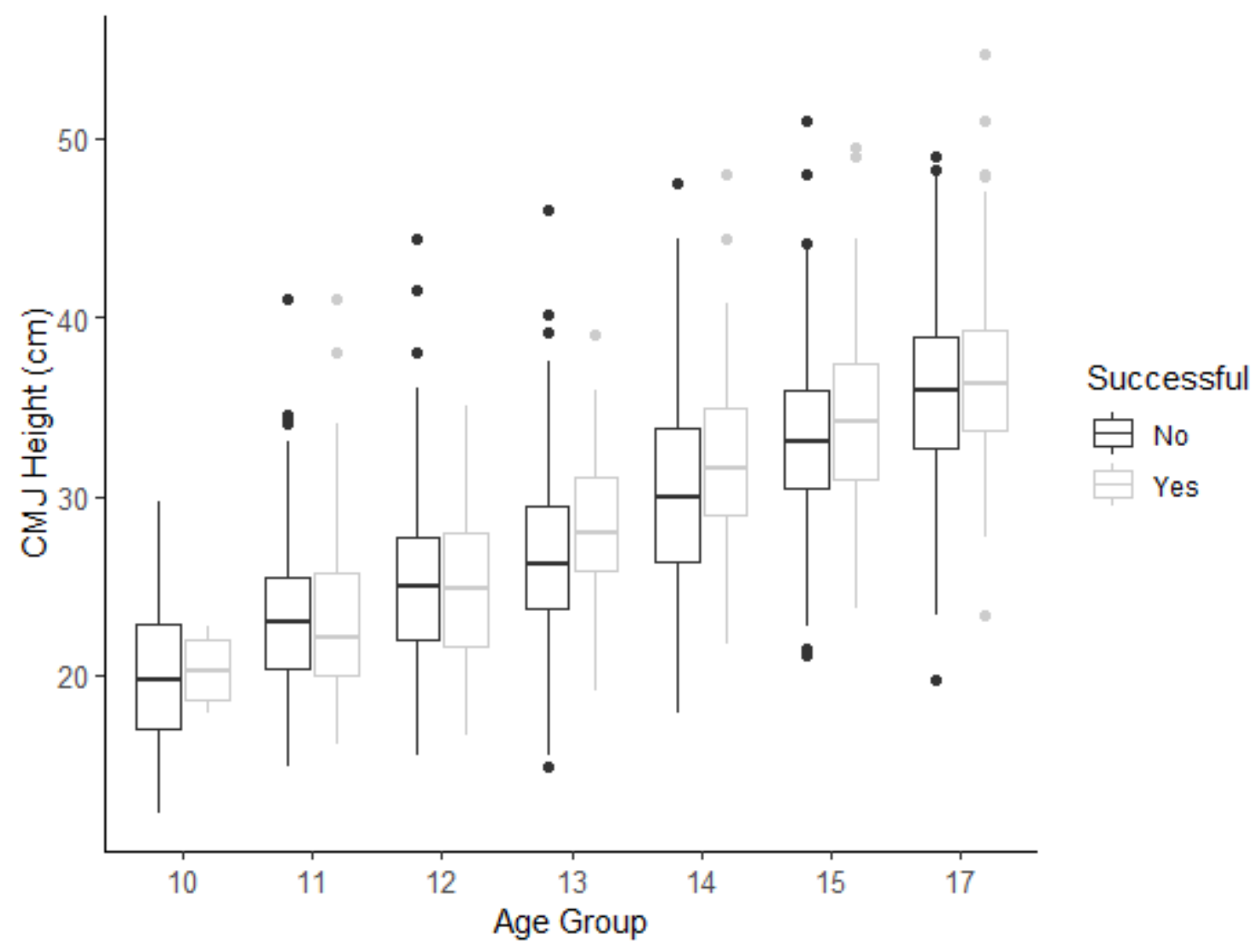

